\title{
Thermal Distortion Analysis of Inflatable Antenna Structures Considering Inflation Gas
}

\author{
Yan Xu1 , Fuling Guan², He Huang², Qian Ye ${ }^{3}$
}

\begin{abstract}
This paper addresses the static distortion and stress analysis of inflatable antenna structures subjected to a thermal load, which is a very important type of load experienced by such structures during service in orbit. Non-linear finite element analysis methods for inflatable structures were formulized, and the thermal stress of thin films was considered. The dynamic relaxation method was used to address the singularity problem of the stiffness matrix for the non-pre-stressed membrane structures. When the thermal load is changing, the state of the inflation gas inside the structure chamber varies, as well as the thermal stress of the membrane material. An iterative algorithm was presented to solve this coupling problem between thermal load, the structures, and inflation gas. The presented algorithm has been implemented into an own finite element code of inflatable structures. The thermal deformation and the stress distribution of a 3.2-m inflatable antenna structure were analyzed to illustrate the feasibility of the numerical method. The results indicated that the change in the state of the inflation gas adversely affects the structural performance, so the inflation gas must be considered.
\end{abstract}

KEYWORDS: Inflatable antenna structures, Thermal distortion analysis, Non-linear FEM, Gas state equation.

\section{INTRODUCTION}

Membrane structures are excellent as architectural and aerospace ones. With respect to the latter, membranes are used to construct inflatable structures, such as inflatable antennas, sunshield structures, sail arrays, Mars explorers, space capsules, etc. Inflatable structures offer many advantages, including low manufacturing cost, light weight and small launch volume.

Thermal loads represent a very important type of load experienced by inflatable antenna structures during service in orbit. To evaluate the performance of such structures, it is necessary to analyze their static deformation and stress distribution under varying external heat flux. To date, the thermal deformation of membrane structures has been investigated through some research and development efforts. The thermomechanical coupling and the effect of thermal disturbance on membrane structures were studied (Wang et al. 2008). A thermal-structural analysis method was described for predicting the stress state in a thin-film membrane of a sunshield subjected to both mechanical and thermal loads (Johnston and Parrish 2003). The structural integrity of a 3-m Ka-Band inflatable/selfrigidizable reflectarray antenna under space thermal conditions was examined (Fang et al. 2003) as well as the temperature distribution, thermal deformation and buckling strength of the inflatable boom. Both theoretical and experimental analyses of a plan membrane reflector under a thermal load were provided (Jenkins and Faisal 2001). The wrinkled deformed shape of the envelope of a stratospheric airship induced by a thermal load was analyzed (Roh et al. 2008). The inflation gas in the internal chamber of these investigated inflatable structures was released

1.Zhejiang University - School of Aeronautics and Astronautics - Department of Aeronautics and Astronautics - Hangzhou - China, 2.Zhejiang University - College of Civil Engineering and Architecture - Department of Civil Engineering - Hangzhou - China. 3.Zhejiang University of Finance and Economics - Dongfang College Department of Engineering Management - Hangzhou - China.

Author for correspondence: Yan Xu | Zhejiang University - School of Aeronautics and Astronautics - Department of Aeronautics and Astronautics | 38 Zheda Rd, Xihu | Postal code: 310027 - Hangzhou - China | Email: xyzs@zju.edu.cn

Received: 02/24/2016 | Accepted: 07/04/2016 
after full deployment; thus, the effect of the residual gas on the performance of the structures was ignored.

For other types of inflatable structures, such as inflatable antenna reflectors (Freeland et al. 1996; Freeland et al. 1997) and lunar habitats (Di Capua et al. 2011; Viscio et al. 2013), the inflation gas in the internal chamber will be kept during service. Therefore, the effect of the residual gas must be considered for thermal deformation analysis. This paper reports a recent effort to develop a theoretical framework for a thermal statics analysis method for inflatable antenna structures considering the variation in the state of the inflation gas in the internal chamber of these structures. An iterative algorithm is presented to solve this coupling problem between thermal load, structure and inflation gas.

The remainder of this paper consists of 4 sections. Inflatable Membrane Mechanics section presents the FEA formulations of inflatable membrane mechanics and the dynamic relaxation method used to address the singularity problem of the stiffness matrix. The iterative algorithm of the thermal statics analysis is described in Thermal Distortion Analysis Method section. The thermal deformation and the stress distribution of a 3.2-m inflatable antenna structure are analyzed in Analysis Results and Discussion section. Finally, Conclusion section provides a few concluding remarks and suggestions for future research.

\section{INFLATABLE MEMBRANE MECHANICS}

The total Lagrange (TL) method is used to formulate the mechanics of membrane structures. The initial configuration $\Omega_{0}$ is selected as the reference configuration in this method. Membrane structures are considered to deform from the known deformed configuration $\Omega_{t}$ to the unknown deformed configuration $\Omega_{t+\Delta t}$, as shown in Fig. 1. Let us consider the deformation from position vector $\mathbf{X}$ to $\mathbf{x}$ with respect to the

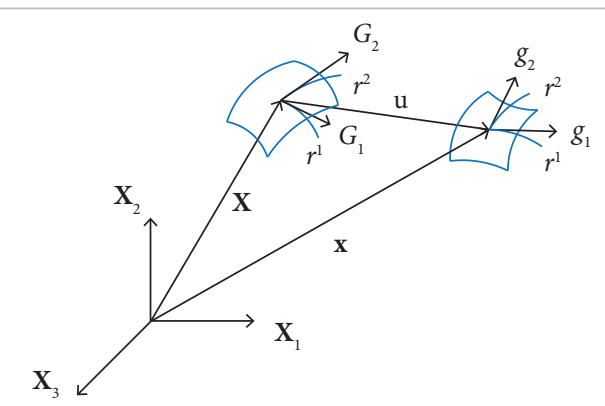

Figure 1. Base vectors of the middle surface. configurations on the surface $\Omega_{0}$ and $\Omega_{t}$, respectively. The following notation is used:

$$
\mathbf{u}=\mathbf{x}-\mathbf{X}
$$

where: $\mathbf{X}$ is the position vector of a material point in the reference configuration; $\mathbf{x}$ is the position vector of a material point in the actual configuration; $\mathbf{u}$ is the deformation vector.

The covariant basis vectors of the surfaces $\Omega_{0}$ and $\Omega_{t}$ are:

$$
\mathbf{G}_{\alpha}=\frac{\partial \mathbf{X}}{\partial r^{\alpha}}, \mathbf{g}_{\alpha}=\frac{\partial \mathbf{x}}{\partial r^{\alpha}}
$$

where: $\alpha=1,2, r^{1}$ and $r^{2}$ are the convective curvilinear coordinates of the surfaces.

The covariant components of the surfaces are defined as:

$$
G_{\alpha \beta}=\mathbf{G}_{\alpha} \cdot \mathbf{G}_{\beta}, g_{\alpha \beta}=\mathbf{g}_{\alpha} \cdot \mathbf{g}_{\beta}
$$

where: $\alpha, \beta=1,2 ; G_{\beta}, g_{\beta}$ are the covariant basis vectors of the surfaces $\Omega_{0}$ and $\Omega_{t}$.

The contravariant basis vectors are obtained as follows:

$$
\mathbf{G}^{\alpha}=G^{\alpha \beta} \mathbf{G}_{\beta}, \mathbf{g}^{\alpha}=g^{\alpha \beta} \mathbf{g}_{\beta}
$$

where: $G^{\alpha \beta}$ and $g^{\alpha \beta}$ are the contravariant components, being $\left[G^{\alpha \beta}\right]=\left[G_{\alpha \beta}\right]^{-1},\left[g^{\alpha \beta}\right]=\left[g_{\alpha \beta}\right]^{-1}$.

For strain and stress measurements, the Green-Lagrange strain tensor $\mathbf{E}$ and the energetically conjugated second PiolaKirchhoff stress tensor ${ }_{0}^{t} \mathbf{S}$ are used. Considering thermal stress, the Green-Lagrange strain tensor $\mathbf{E}$ is given by:

$\mathbf{E}=\frac{1}{2}\left({ }_{0}^{t} \mathbf{X}_{0}^{T}{ }_{0}^{t} \mathbf{X}-\mathbf{I}\right)-\boldsymbol{\alpha} \Delta T=\frac{1}{2}\left(\mathbf{g}_{\alpha \beta}-\mathbf{G}_{\alpha \beta}\right) \mathbf{G}^{\alpha} \otimes \mathbf{G}^{\beta}-\boldsymbol{\alpha} \Delta T$

where: ${ }_{0}^{t} \mathbf{X}$ is the deformation gradient, which links the reference configuration to the deformed one; $\mathbf{I}$ is the unit tensor; $\gamma$ is the thermal expansion coefficient; $\Delta T$ is the change in temperature.

The second Piola-Kirchhoff stress tensor is expressed in terms of the Green-Lagrange strain tensor by the constitutive law for an isotropic and linear elastic material:

$$
{ }_{0}^{t} \mathbf{S}=\mathbf{C}:{ }_{0}^{t} \mathbf{E}
$$


where: $\mathbf{C}$ is the elastic matrix of the material; ${ }_{0}^{t} \mathbf{E}$ is the Green-Lagrange strain tensor.

The formula relating the Cauchy stress tensor to the second Piola-Kirchhoff stress tensor is:

$$
{ }_{0}^{t} \mathbf{S}=\mathbf{J}_{0}^{t} \mathbf{X}^{-1} \cdot{ }_{t}^{t} \boldsymbol{\tau} \cdot{ }_{0}^{t} \mathbf{X}^{-T}
$$

where: $\mathbf{J}$ is the determinant of the Jacobian matrix; ${ }_{t}^{t} \boldsymbol{\tau}$ is the Cauchy stress tensor.

The weak form of the equilibrium equation is attained by the principle of virtual work, which states that the sum of internal and external works vanishes at equilibrium:

$$
\int_{0_{V}}{ }_{0}^{t+\Delta t} \mathbf{S}_{\alpha \beta} \delta\left({ }_{0}^{t+\Delta t} \mathbf{E}_{\alpha \beta}\right) d^{0} V={ }^{t+\Delta t} \delta \mathbf{R}
$$

where: ${ }^{0} V$ is the volume of the structure; ${ }^{t+\Delta t} \delta \mathbf{R}$ is the work done by the external loads.

The stiffness matrix of the membrane element includes two parts: the initial deformation stiffness matrix and the geometric stiffness matrix. The initial deformation stiffness matrix $\mathbf{K}_{L}$ can be obtained as:

$$
\begin{aligned}
& \mathbf{K}_{L}=\int_{\sigma_{V}}\left({ }_{0}^{t} \mathbf{B}_{L}^{T} \cdot{ }_{0} \mathbf{C} \cdot{ }_{0}^{t} \mathbf{B}_{L}\right) d^{0} V \\
& \text { where: }{ }_{0}^{t} \mathbf{B}_{L}=\left\lfloor\begin{array}{llll}
{ }_{0}^{t} \mathbf{B}_{L}^{1} & { }_{0}^{t} \mathbf{B}_{L}^{2} & \ldots & { }_{0}^{t} \mathbf{B}_{L}^{n}
\end{array}\right\rfloor \\
& { }_{0}^{t} \mathbf{B}_{L}^{n}=\left[\begin{array}{cc}
\left({ }^{t} \mathbf{g}_{1}\right)_{1} \frac{\partial N^{n}}{\partial r^{1}} & \left({ }^{t} \mathbf{g}_{1}\right)_{2} \frac{\partial N^{n}}{\partial r^{1}} \\
\left({ }^{t} \mathbf{g}_{2}\right)_{1} \frac{\partial N^{n}}{\partial r^{2}} & \left({ }^{t} \mathbf{g}_{2}\right)_{2} \frac{\partial N^{n}}{\partial r^{2}} \\
\left.{ }^{t} \mathbf{g}_{1}\right)_{1} \frac{\partial N^{n}}{\partial r^{2}}+\left({ }^{t} \mathbf{g}_{2}\right)_{1} \frac{\partial N^{n}}{\partial r^{1}} & \left({ }^{t} \mathbf{g}_{1}\right)_{2} \frac{\partial N^{n}}{\partial r^{2}}+\left({ }^{t} \mathbf{g}_{2}\right)_{2} \frac{\partial N^{n}}{\partial r^{1}}
\end{array}\right. \\
& \left({ }^{t} \mathbf{g}_{1}\right)_{3} \frac{\partial N^{n}}{\partial r^{1}} \\
& \left({ }^{t} \mathbf{g}_{2}\right)_{3} \frac{\partial N^{n}}{\partial r^{2}} \\
& \left({ }^{t} \mathbf{g}_{1}\right)_{3} \frac{\partial N^{n}}{\partial r^{2}}+\left({ }^{t} \mathbf{g}_{2}\right)_{3} \frac{\partial N^{n}}{\partial r^{1}}
\end{aligned}
$$

where: $\left({ }^{t} \mathbf{g}_{\alpha}\right)_{i}$ is the component of ${ }^{t} \mathbf{g}_{\alpha}$ according to the global coordinate vector $\mathbf{e}_{i} ;{ }_{0} \mathbf{C}$ is the elasticity tensor in the configuration $\Omega_{0} ; N^{n}$ is the interpolation function according to node $n$ in the element.

The geometric stiffness matrix $\mathbf{K}_{N L}$ can be obtained from:

$$
\mathbf{K}_{N L}=\int_{\sigma_{V}}\left({ }_{0}^{t} \mathbf{B}_{N L}^{T} \cdot{ }_{0}^{t} \mathbf{S} \cdot{ }_{0}^{t} \mathbf{B}_{N L}\right) d^{0} V
$$

$$
\begin{gathered}
\text { where: }{ }_{0}^{t} \mathbf{B}_{N L}=\left[\begin{array}{lllll}
{ }_{0}^{t} \mathbf{B}_{N L}^{1} & { }_{0}^{t} \mathbf{B}_{N L}^{2} & \mathrm{~L} & { }_{0}^{t} \mathbf{B}_{N L}^{n}
\end{array}\right] \\
{ }_{0}^{t} \mathbf{B}_{N L}^{n}=\left[\begin{array}{cccccc}
\frac{\partial N^{n}}{\partial r^{1}} & 0 & 0 & \frac{\partial N^{n}}{\partial r^{2}} & 0 & 0 \\
0 & \frac{\partial N^{n}}{\partial r^{1}} & 0 & 0 & \frac{\partial N^{n}}{\partial r^{2}} & 0 \\
0 & 0 & \frac{\partial N^{n}}{\partial r^{1}} & 0 & 0 & \frac{\partial N^{n}}{\partial r^{2}}
\end{array}\right]^{T}
\end{gathered}
$$

The formulas used to analyze inflatable structures do not feature a pre-stress of the material at the initial configuration. If the bending stiffness of the thin-film material is ignored, the geometric stiffness matrix $\mathbf{K}_{N L}$ is zero, and the values in the initial stiffness matrix are small. The process of FEM iterative calculation cannot be successfully employed because of the singularity of the stiffness matrix. The dynamic relaxation method is presented to solve this problem. Through this method, the static problem is transformed into a quasi-dynamic problem. The deformation of a non-pre-stressed membrane subjected to thermal loads is analyzed by the iterative calculation. The equilibrium formula is rewritten as follows:

$$
\mathbf{Q}(u)=\mathbf{F}
$$

where: $\mathbf{Q}$ is the internal force vector; $\mathbf{F}$ is the external force vector; $u$ is the displacement vector of the nodes.

The N-R method is applied to solve the equilibrium formula:

$$
\mathbf{K}\left({ }^{k} \mathbf{u}\right) *\left({ }^{k+1} \mathbf{u}-{ }^{k} \mathbf{u}\right)=\mathbf{F}-\mathbf{Q}\left({ }^{k} \mathbf{u}\right)
$$

where the tangent stiffness matrix is:

$$
\mathbf{K}\left({ }^{k} \mathbf{u}\right)=\frac{\partial \mathbf{Q}\left({ }^{k} \mathbf{u}\right)}{\partial \mathbf{u}}
$$

When the thin-film material is not pre-stressed at the initial configuration, the stiffness matrix is singular and Eq. 12 cannot be solved. The static problem is equivalent to the quasi-dynamic problem described by the following quation:

$$
\mathbf{D} \dot{\mathbf{u}}+\mathbf{Q}(\mathbf{u})=\mathbf{F}
$$

where: $\mathbf{D}$ is the hypothetical damping term.

After the iterative calculation convergence, the velocity term $\dot{\mathbf{u}}$ tends to zero. The previous equation is transformed into Eq. 11. In one time step, the velocity term $\dot{\mathbf{u}}$ is: 


$$
\dot{\mathbf{u}}=\frac{{ }^{k+1} \mathbf{u}-{ }^{k} \mathbf{u}}{\Delta t}
$$

where: $\Delta t$ is the hypothetical iterative time step.

Equation 15 is substituted into the N-R method equation, then:

$$
\left[\mathbf{K}\left({ }^{k} \mathbf{u}\right)+\frac{\mathbf{D}}{\Delta t}\right] *\left({ }^{k+1} \mathbf{u}-{ }^{k} \mathbf{u}\right)=\mathbf{F}-\mathbf{Q}\left({ }^{k} \mathbf{u}\right)
$$

The iterative calculation process is completed when the previous equation and the equilibrium state subjected to the loads are calculated:

$$
{ }^{k+1} \mathbf{u}-{ }^{k} \mathbf{u}=\dot{\mathbf{u}} \Delta t
$$

The hypothetical damping term in Eq. 14 is:

$$
\mathbf{D}={ }^{k} \mu \mathbf{I}
$$

where: ${ }^{k} \mu$ is the attenuation coefficient.

To accelerate the iterative calculation, ${ }^{k} \mu$ can be determined as:

$$
{ }^{+1} \mu={ }^{k} \mu \frac{\left\|{ }^{k} \Delta \mathbf{u}\right\|}{\left\|{ }^{k-1} \Delta \mathbf{u}\right\|}
$$

where: ${ }^{k} \Delta \mathbf{u}$ is the increment in the node displacement vector in $k^{\text {th }}$ iterative step, and the attenuation coefficient ${ }^{k} \mu$ of the first step is manually input.

\section{THERMAL DISTORTION ANALYSIS}

The equation of state for an ideal gas is:

$$
P V=n R T
$$

where: $V, P$, and $T$ are the volume, pressure, and temperature, respectively, of the inflation gas.

For the internal chamber of the inflatable structure, the leaking of the inflation gas is ignored. Therefore, the gas undergoes a transition from the initial state $\left(P_{0}, V_{0}, T_{0}\right)$ to the final one $\left(P_{1}, V_{1}, T_{1}\right)$ and yields:

$$
\frac{V_{1} P_{1}}{T_{1}}=\frac{V_{0} P_{0}}{T_{0}}=k
$$

where: $k=n R$, which only depends on the numerator of the inflation gas state equation.

When the leaking of the inflation gas is ignored, $k$ is a constant value. If the environmental temperature changes to $T_{1}$, from the equation of state for an ideal gas,

$$
P_{1}=\frac{k T_{1}}{V_{1}}
$$

As indicated by Eq. 22, pressure and volume are inversely proportional. As the environmental temperature increases or decreases, the deformation of the membrane surface and the equilibrium state of the inflation gas vary synchronously. A new equilibrium state is reached when the constant value $k$ reverts to the initial value. An iterative algorithm is required to solve this coupling problem and to calculate this new equilibrium state.

\section{ITERATIVE ALGORITHM}

The purpose of this section is to present an iterative algorithm for calculating the new equilibrium state of the gas in the inflatable structure chamber after the environmental temperature changes, where the pressure and volume of the gas are analyzed.

As shown in Fig. 2, when the environmental temperature changes from $T_{0}$ to $T_{1}$, the pressure and volume of the inflation gas change from their initial values to $P_{1}$ and $V_{1}$, respectively, where $P_{\text {max }}=k T_{1} / V_{0} ; V_{\text {max }}=k T_{1} / P_{0}$. When the temperature changes to $T_{2}$, the pressure and volume of the inflation gas change from their initial values to $P_{2}$ and $V_{2}$, respectively, where $P_{\text {min }}=k T_{2} / V_{0} ; V_{\text {min }}=k T_{2} / P_{0}$.

From the initial state $V_{0}, P_{0}, T_{0}$ of the inflation gas to the new equilibrium state $V_{1}, P_{1}, T_{1}$ (or $V_{2}, P_{2}, T_{2}$ ), there are $n$ increment steps in the calculation process. When the temperature changes to $T_{1}$, the volume of the gas is assumed to be constant at first. Thus, according to the state equation of a constant change in volume, the pressure changes to

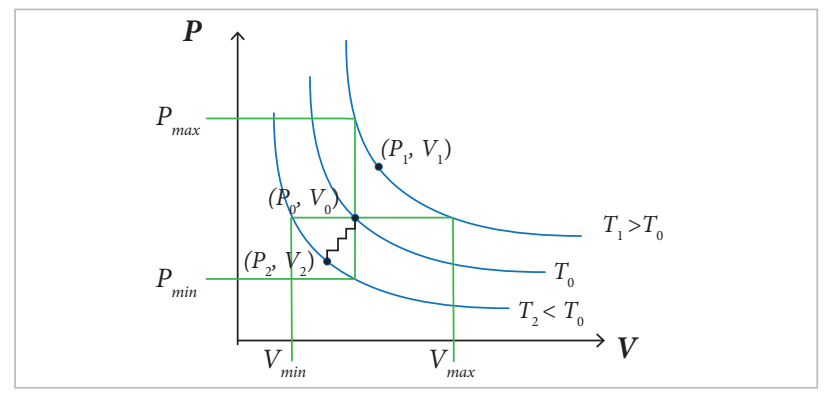

Figure 2. Pressure-volume curve of the inflation gas. 
$P_{1}^{*}=T_{1} P_{0} / T_{0}$. Therefore, the increase in the gas pressure in the first incremental step is $\Delta P_{1}=\left(P_{1}^{*}-P_{0}\right) / n$. The total pressure after this step is $P_{1}=P_{0}+\Delta P_{1}$. Thus, the thinfilm material deforms under this pressure. The deformed configuration and the new volume of the structure chamber $V_{1}$ are determined by FEM analysis. Based on the equation of state for an ideal gas, the new pressure is obtained: $P_{2}^{*}=\left(P_{0} V_{0} T_{1}\right) /\left(T_{0} V_{1}\right)$. The increase in pressure in the second incremental step is $\Delta P_{2}=\left(P_{2}^{*}-P_{1}\right) /(n-1)$. The total pressure after this step is $P_{2}=P_{1}+\Delta P_{2}$. The deformed configuration and the new volume of the structure chamber $V_{2}$ are analyzed again. Further iteration is performed until the increase in pressure in each incremental step is very small.

\section{ENCLOSED VOLUME OF STRUCTURE CHAMBER}

The volume of the structure chamber must be calculated in the iterative algorithm. The method for calculating the volume of the chamber is presented in this section. The structure's surface is modeled by a mesh composed of triangular elements. The volume of the chamber is equal to the sum of the volumes of the triangular prisms covered by the corresponding triangular elements. The volume of one triangular prism is shown in Fig. 3. The underside of each triangular prism is on the $x y$ plane in the global coordinate system. The volume of each triangular prism is:

$$
\begin{aligned}
& V=\frac{1}{6} \mid\left(x_{j}-x_{i}\right)\left(y_{k}-y_{i}\right)- \\
& -\left(x_{k}-x_{i}\right)\left(y_{j}-y_{i}\right) \mid\left(z_{i}+z_{j}+z_{k}\right)
\end{aligned}
$$

The absolute value sign guarantees that the volume value is always positive, whether the nodes $i, j$, and $k$ are numbered clockwise or counterclockwise. When the three nodes $i, j$,

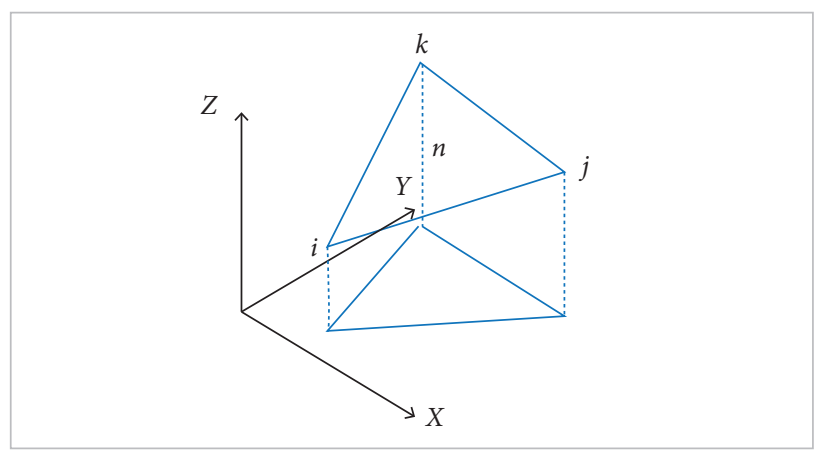

Figure 3. Chamber volume of a triangular prism. and $k$ of the $n$ element deform from a configuration $X_{n}$, the increase in the value of the node displacement vector is $\Delta d_{n}$ and the increase in the triangular prism volume is $\Delta V_{n}$. The relationship between these 2 incremental values is:

$$
\Delta V_{n}=V_{n}\left(\mathbf{X}_{n}+\mathbf{u}_{n}\right)-V_{n}\left(\mathbf{X}_{n}\right)=\mathbf{b}_{n} \mathbf{u}_{n}
$$

where:

$$
\begin{aligned}
& \mathbf{b}_{n}=\frac{1}{6}\left[\begin{array}{lllllllll}
b_{n}^{1} & b_{n}^{2} & b_{n}^{3} & b_{n}^{4} & b_{n}^{5} & b_{n}^{6} & b_{n}^{7} & b_{n}^{8} & b_{n}^{9}
\end{array}\right] \\
& b_{n}^{1}=\left(y_{j}-y_{k}\right)\left(z_{i}+z_{j}+z_{k}\right) \text {, } \\
& b_{n}^{2}=\left(x_{k}-x_{j}\right)\left(z_{i}+z_{j}+z_{k}\right) \text {, } \\
& b_{n}^{3}=\left(x_{j}-x_{i}\right)\left(y_{k}-y_{i}\right)-\left(x_{k}-x_{i}\right)\left(y_{j}-y_{i}\right) \\
& b_{n}^{4}=\left(y_{k}-y_{i}\right)\left(z_{i}+z_{j}+z_{k}\right) \text {, } \\
& b_{n}^{5}=\left(x_{i}-x_{k}\right)\left(z_{i}+z_{j}+z_{k}\right) \text {, } \\
& b_{n}^{6}=\left(x_{j}-x_{i}\right)\left(y_{k}-y_{i}\right)-\left(x_{k}-x_{i}\right)\left(y_{j}-y_{i}\right) \\
& b_{n}^{7}=\left(y_{i}-y_{j}\right)\left(z_{i}+z_{j}+z_{k}\right) \text {, } \\
& b_{n}^{8}=\left(x_{j}-x_{i}\right)\left(z_{i}+z_{j}+z_{k}\right) \text {, } \\
& b_{n}^{9}=\left(x_{j}-x_{i}\right)\left(y_{k}-y_{i}\right)-\left(x_{k}-x_{i}\right)\left(y_{j}-y_{i}\right) \\
& \mathbf{u}_{n}=\left[\begin{array}{lllllllll}
d x_{i} & d y_{i} & d z_{i} & d x_{j} & d y_{j} & d z_{j} & d x_{k} & d y_{k} & d z_{k}
\end{array}\right]^{T}
\end{aligned}
$$

This equation describes the change in the chamber volume with the deformation of the surface.

\section{RESULTS AND DISCUSSION}

In this section, the thermal distortion analysis of an inflatable antenna structure is performed to illustrate the feasibility of the numerical method. A 3.2-m ground demonstration space antenna structure was designed and manufactured (Xu and Guan 2012). The thermal distortion analysis method was applied to the inflatable antenna structure, but the high- and low-temperature experiments have not been performed yet to validate these results. The ground demonstration model is shown in Fig. 4. The aperture of the inflatable reflector is $3.2-\mathrm{m}$, and the ratio between the focus length and aperture is 0.5 . The designed inflatable pressure is $20 \mathrm{~Pa}$. The membrane material is Kapton $\mathbb{}$ VN (DuPont ${ }^{\mathrm{TM}}$ ). The elastic modulus is $2.5 \mathrm{GPa}$, the Poisson's ratio is 0.340 and the membrane thickness is $0.025 \mathrm{~mm}$. The coefficients of thermal expansion of the thin-film material in each temperature range are shown in Table 1. 


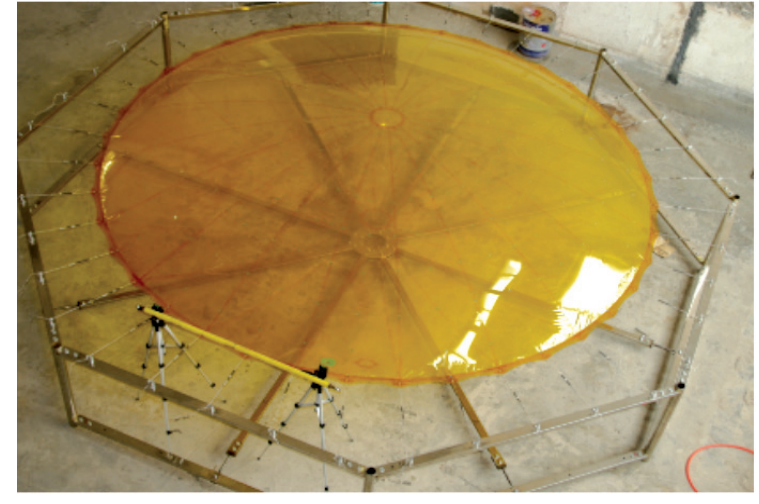

Figure 4. Ground demonstration model (Xu and Guan 2012).

Table 1. Thermal expansion coefficients of Kapton® VN (DuPont $\left.{ }^{T M}\right)$.

\begin{tabular}{l|l} 
Temperature range & Thermal expansion coefficient
\end{tabular} [ $\mathrm{C}]$

$30-100$

17

$100-200$

32

$200-300$

40

$300-400$

44

The inflatable antenna structure consists of a reflective surface, canopy, tension/adjustment cable system and support structure. The reflective surface and the canopy were assembled individually by fastening the planar membrane gores together. The reflective surface and the canopy were then glued together along their circumferences to form an envelope structure to contain the inflation fluid (i.e. ambient air in our case). The envelope structure was mounted onto the support structure by the tension/adjustment cable system.

The iterative algorithm presented in the last section was employed to analyze the thermal static deformation and stress of this inflatable antenna structure. Considering structural symmetry, the thermal static deformation of reflector was calculated. The reflector surface was modeled by 360 triangular elements, and each tension/adjustment cable was meshed by a single element. The movement of each circumference node of the envelope structure along the $z$ direction was fixed, as was that of the nodes on the support structure along all directions. Top and side views of the numerical model are shown in Fig. 5.

Because the size of this demonstration model is small, the temperature gradient on the structure's surface is ignored. The environmental temperature of the inflatable structure

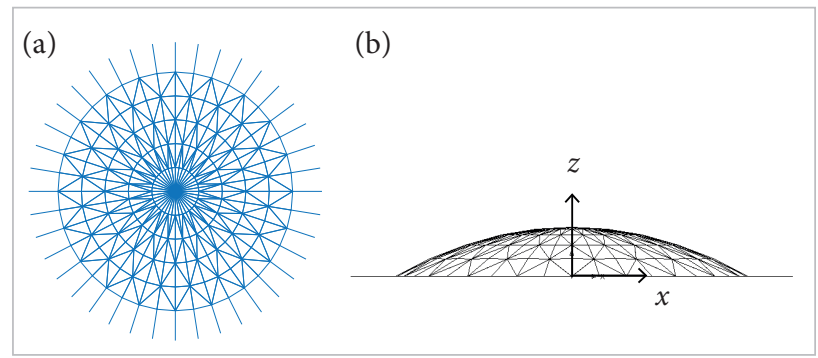

Figure 5. Numerical model of the inflatable antenna. (a) Top view; (b) Side view.

changes from 30 to $10^{\circ} \mathrm{C}$. Two load cases were defined and analyzed to investigate the effect of the inflation gas.

- Load case 1: Only the thermal deformation of the membrane material was considered; the pressure in the chamber was fixed to the designed pressure, $20 \mathrm{~Pa}$.

- Load case 2: The thermal deformation of the membrane material and the change in the state of the inflation gas were considered to occur synchronously. After the environmental temperature changed, the gas pressure was no longer equal to the designed pressure.

These two load cases were analyzed. The total number of incremental steps was $n=50$. The results of the analysis, the $z$ coordinate of the vertex and the RMS of the reflective surface are shown in Table 2.

Table 2. The numerical results of the reflective surface.

\begin{tabular}{|c|c|c|}
\hline & $\begin{array}{c}\text { Z coordinate of } \\
\text { the vertex }[\mathrm{m}]\end{array}$ & $\begin{array}{c}\text { RMS of the reflective } \\
\text { surface }(\mathrm{mm})\end{array}$ \\
\hline $\begin{array}{c}\text { Initial } \\
\text { configuration }\end{array}$ & 0.4268 & 0.1295 \\
\hline Load case 1 & 0.4254 & 0.1883 \\
\hline Load case 2 & 0.4252 & 0.5713 \\
\hline
\end{tabular}

The numerical results for the second load case, and the curve relating pressure $P$ and volume $V$ for each incremental step are shown in Fig. 6. Through iterative analysis, the initial gas state $\left(V_{0}, P_{0}\right)$ in the curve changes to the final one $\left(V_{2}, P_{2}\right)$, where $P_{2}=18.35 \mathrm{~Pa}$ and $V_{2}=1.6853 \mathrm{~m}^{3}$.

The gas constant value $k$ changes though the iterative analysis process (Fig. 7). At first, the $k$ value greatly deviates from the initial one, $0.1232 \mathrm{Nm} /{ }^{\circ} \mathrm{C}$. Then, the $k$ value tends to the initial one, reaching $0.1132 \mathrm{Nm} /{ }^{\circ} \mathrm{C}$.

After the environmental temperature changes, the maximum displacement of the nodes $(-1.7 \mathrm{~mm})$ occurs along the top 
of the reflector surface. The maximum stress zone of the membrane material occurs along the boundary of the canopy. The maximum first principle stress is $1.56 \mathrm{MPa}$, the maximum second principle stress is $1.32 \mathrm{MPa}$, and the tension stress of the cables is $12.56 \mathrm{MPa}$.

Based on the analytical results, it can be concluded that the effect of the thermal stress of the thin-film material on the deformation of the structure is not very great. But the change in the state of the inflation gas adversely affects the reflector precision, and the inflation gas must be considered in evaluating the structural performance of the inflatable structures. After the environmental temperature changes, the final gas pressure is not equal to the designed pressure.

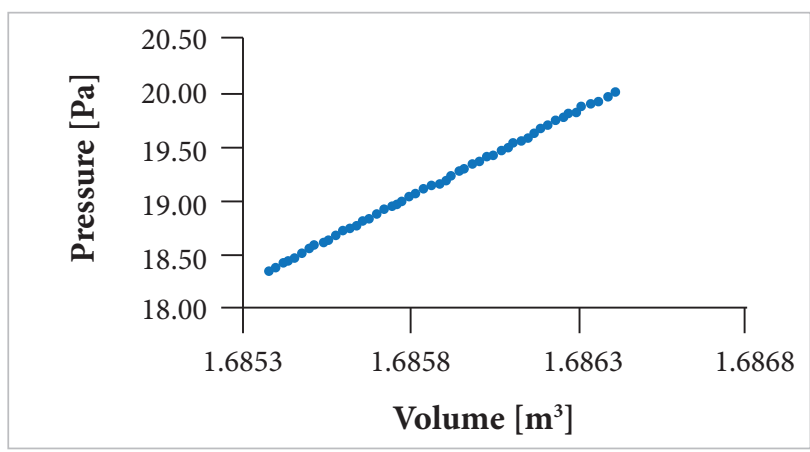

Figure 6. Pressure-volume curve of the reflector structure.

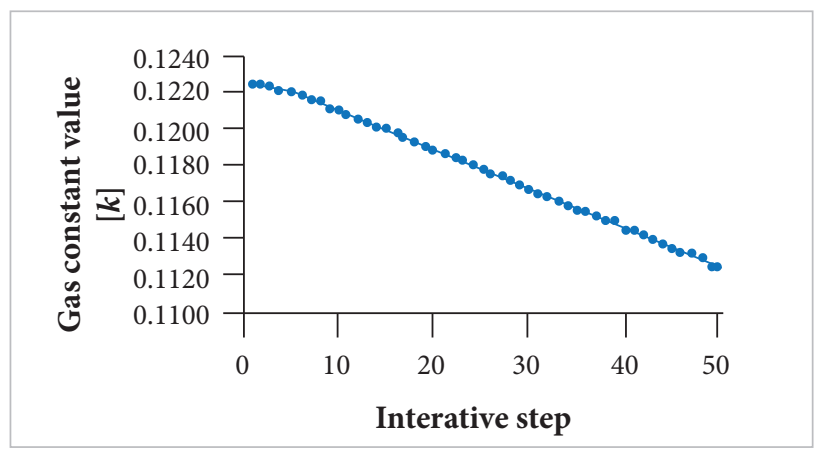

Figure 7. Gas constant value change.

\section{CONCLUSION}

A thermal statics analysis method for inflatable antenna structures considering the variation in the state of the inflation gas was investigated. An iterative algorithm was presented to solve the coupling problem between thermal load, structural deformation, and inflation gas. The dynamic relaxation method was used to address the singularity problem of the stiffness matrix for the non-pre-stressed membrane structures. The analytical results indicated that the effect of the thermal stress of the membrane material on the distortion distribution and stress distribution of the structure is not very great, although the change in the state of the inflation gas adversely affects the final configuration. The thermal deformation of the inflatable antenna structures is large, and it is necessary to investigate the thermal and inflation pressure control system. As the antenna moves in and out of the Sun, the inflatable gas may be vented and refilled. Thus, the present study will provide a reference for the controller design.

\section{ACKNOWLEDGEMENTS}

This paper was supported by the National Natural Science Foundation of China (Grant No. 11402229) and the Natural Science Foundation of Zhejiang Province (Grant No. LQ14A020003).

\section{AUTHOR'S CONTRIBUTION}

$\mathrm{Xu} \mathrm{Y}$ and Guan F conceived the idea and co-wrote the main text. Xu Y and Huang H performed the numerical simulation and experimental works. Ye Q prepared the figures and formulas. All authors discussed the results and commented on the manuscript.

\section{REFERENCES}

Di Capua M, Akin DL, Davis K (2011) Design, development, and testing of an inflatable habitat element for NASA lunar analogue studies. Proceedings of the 41st International Conference on Environmental Systems; Portland, USA.

Fang $H$, Lou M, Huang J, Quijiano U, Hsia LM (2003) Thermal distortion analyses of a three-meter inflatable reflectarray antenna. Proceedings of the 44th AIAA/ASME/ASCE/AHS/ASC Structures,
Structural Dynamics, and Materials Conference; Norfolk, USA.

Freeland RE, Bilyeu GD, Veal GR (1996) Development of flight hardware for a large, inflatable-deployable antenna experiment. Acta Astronaut 38(4-8):251-260. doi: 10.1016/0094-5765(96)00030-6

Freeland RE, Bilyeu GD, Veal GR, Steiner MD, Carson DE (1997) Large inflatable deployable antenna flight experiment results. Acta Astronaut 
41(4-10):267-277. doi: 10.1016/S0094-5765(98)00057-5

Jenkins CH, Faisal SM (2001) Thermal load effects on precision membranes. J Spacecraft Rockets 38(2):207-211. doi: $10.2514 / 2.3671$

Johnston J, Parrish K (2003) Thermal-structural analysis of sunshield membranes. Proceedings of the 2003 AIAA Structures, Structural Dynamics, and Materials Conference; Norfolk, USA.

Roh JH, Lee HG, Lee I (2008) Thermoelastic behaviors of fabric membrane structures. Adv Compos Mater 17(4):319-332. doi: 10.1163/156855108X385285
Viscio MA, Viola N, Gargioli E, Vallerani E (2013) Conceptual design of a habitation module for a deep space exploration mission. Proc IME G J Aero Eng 227(9):1389-1411. doi: 10.1177/0954410012457292

Wang XY, Sulik C, Zheng WP, Hu YR (2008) Thermo-mechanical analysis of thin membranes and application in active flatness control design. Proceedings of the Industrial and Commercial Applications of Smart Structures Technologies 2008; San Diego, USA.

Xu Y, Guan FL (2012) Structure design and mechanical measurement of inflatable antenna. Acta Astronaut 76:13-25. doi: 10.1016/j. actaastro.2012.02.005 